\title{
Posttranslational Truncation of E-Cadherin and Significance for Tumour Progression
}

\author{
Joanne Masterson Shirley O'Dea \\ Institute of Immunology, Biology Department, National University of Ireland Maynooth, Maynooth, Ireland
}

\section{Key Words}

E-cadherin $\cdot$ E-cadherin truncation $\cdot$ Matrix

metalloproteinase $\cdot$ Tumour progression

\begin{abstract}
Stable intraepithelial adhesion complexes are essential for the maintenance of epithelial integrity. Alterations in these complexes are key events in the development and progression of many diseases. One of the major proteins involved in maintaining epithelial cell-cell adhesion is the cell-adhesion junction protein E-cadherin, a member of the cadherin family of transmembrane adhesion proteins. E-cadherin is involved in many cellular processes including morphogenesis, adhesion, recognition, communication and oncogenesis. Inactivation of its adhesive properties is often a key step in tumour progression and metastasis, leading to its recent description as a tumour suppressor gene. Mutations of the Ecadherin gene $\mathrm{CDH} 1$ in gastric and mammary cancers have
\end{abstract}

\section{Abbreviations used in this paper}

E-cad ${ }^{100} \quad 100-k D a$ E-cadherin fragment

EMT epithelial-mesenchymal transition

HAV histadine alanine valine

HGF/SF hepatocyte growth factor/scatter factor

MMP matrix metalloproteinase

sE-cad soluble E-cadherin

SL-1 stromelysin-1 been well documented and reports of transcriptional repression during tumour progression are increasing. This review examines the role of posttranslational truncation of $\mathrm{E}$ cadherin in cancer cells focusing on implications for tumour progression. The various proteins involved in the directed cleavage of E-cadherin and consequences of these truncations are discussed.

Copyright $\odot 2007$ S. Karger AG, Basel

\section{E-Cadherin-Mediated Cell Adhesion}

Classical adherens junctions, tight junctions, gap junctions and desmosomal junctions are responsible for controlling secure cell-to-cell contact in epithelia, maintaining epithelial cell polarity and tissue integrity. E-cadherin (also known as uvomorulin, cell-CAM $120 / 80$ or LCAM) is a member of the cadherin family of transmembrane adhesion proteins that form adherens junctions [Angst et al., 2000]. E-cadherin is a $124-\mathrm{kDa}$, epithelialspecific glycoprotein involved in many cellular processes including adhesion, recognition, signalling, communication, morphogenesis and oncogenesis [Ivanov et al., 2001]. E-cadherin homodimers are located at the cell membrane where they interact with E-cadherin dimers on adjacent cells in a calcium-dependent manner [Ivanov et al., 2001]. Interaction of E-cadherin with the actin cytoskeleton is mediated by the cytoplasmic proteins $\alpha$-, $\beta$ - and $\gamma$-catenin resulting in stabilized adherens complexes and polarized epithelial cells [Hirano et al., 1987; Ozawa et al., 1989; Noritake et al., 2004]. Alterations in cell-cell contacts, in-
Tel. +3531708 3242, Fax +3531708 6337, E-Mail Joanne.Masterson@nuim.ie 
cluding those that involve E-cadherin, occur to facilitate normal physiological processes such as morphogenesis, epithelial-mesenchymal transition (EMT) during organogenesis, cell division and apoptosis [Reinsch and Karsenti, 1994; Ivanov et al., 2001; Steinhusen et al., 2001; Zhu et al., 2001; Li et al., 2003; Shen and Kramer, 2004]. Disruption of cell-cell contacts and cell migration are key events in EMT and are increasingly linked with tumour progression, invasion and dissemination. While these may be critical to early stages of tumour progression, good evidence is emerging to indicate that re-expression of E-cadherin/re-epithelialization at the site of metastasis favours growth of the secondary tumour [see Chaffer et al., 2006, this volume], suggesting a dynamic regulation throughout the metastasis process. Improved understanding of underlying mechanisms would contribute to identification of prognostic markers and novel therapeutic targets.

The regulation of cell adhesion complex proteins, and specifically E-cadherin, during disease processes has become the focus of intensive research. Investigations into reduced E-cadherin function in cancer have included hereditary mutational analysis, promoter hypermethylation analysis, transcriptional repression analysis and posttranslational modification studies. Posttranslational truncation of E-cadherin and subsequent disruption of cell adhesion appear to facilitate tumour dissemination. Furthermore, the resulting increase in cytoplasmic $\beta$ catenin levels can enhance proliferation via the wnt signalling pathway [Korinek et al., 1997]. In this paper, we review the evidence that posttranslational truncation of E-cadherin is associated with tumour progression.

\section{Posttranslational Truncation of E-Cadherin}

Posttranslational modification of E-cadherin occurs during normal maturation of the protein. E-cadherin is synthesized as a $135-\mathrm{kDa}$ inactive precursor protein [Peyrieras et al., 1983]. Maturation in the Golgi network involves proteolytic cleavage of pro-E-cadherin near the Nterminus at a specific recognition sequence [Arg/Lys$(\mathrm{X})_{\mathrm{n}}$-Lys/Arg-Arg] $(\mathrm{n}=0,2,4$ or 6$)$ by a subtilisin-like proprotein convertase (fig. 1a). Once processed the functional mature E-cadherin protein is translocated to the cell membrane (fig. 1b). While pro-E-cadherin can integrate into the cell membrane, it lacks adhesive ability, indicating that proteolytic processing is essential for the adhesive function of E-cadherin [Ozawa and Kemler, 1990].
The first alternative form of E-cadherin described was a soluble $80-\mathrm{kD}$ a protein, gp80, which was subsequently found to be soluble E-cadherin (sE-cad) (fig. 1c). This protein is constitutively released into culture medium by MCF-7 human mammary carcinoma cells. sE-cad-containing conditioned medium inhibits aggregation of cultured epithelial cells and prevents cell compaction at the eight-cell stage of mouse embryogenesis [Damsky et al., 1983; Wheelock et al., 1987]. This truncated form of Ecadherin may function as a pseudo-ligand that blocks normal E-cadherin interactions, resulting in reduced adhesion. Elevated levels of sE-cad have also been detected in vivo in serum and urine of cancer patients, suggesting a link between E-cadherin truncation and tumourigenesis [Katayama et al., 1994; Banks et al., 1995; Griffiths et al., 1996; Gofuku et al., 1998; Protheroe et al., 1999; Rashid et al., 2001].

Studies involving the matrix metalloprotease (MMP) stromelysin-1 (SL-1) indicate that $\mathrm{sE-cad}$ occurs as a result of protease-mediated truncation of E-cadherin [Lochter et al., 1997]. Lochter et al. detected soluble 95and $80-\mathrm{kDa}$ fragments that react with the ECCD-2 antiE-cadherin antibody following overexpression of SL-1 in SCp2 mammary epithelial cells. Appearance of these fragments was inhibited by the GM6001 general MMP inhibitor. A $40-\mathrm{kDa}$ ECCD-2-reactive fragment is also detected regardless of SL-1 presence. SL-1 expression induces cell scattering, an invasive phenotype and expression of a mesenchymal-like cytoskeleton with decreased cytokeratin and increased vimentin expression. While increased keratinocyte growth factor mRNA expression also occurs, recombinant keratinocyte growth factor alone is not enough to induce the phenotypic changes induced by SL-1. The MMP family of proteases regulates ductal morphogenesis, apoptosis and neoplastic progression in mammary epithelial cells [Lochter et al., 1997]. MMPs were traditionally thought to contribute to tumourigenesis solely via degradation of the extracellular matrix, thereby facilitating invasion during tumour progression. It now appears, however, that SL-1 activity has multiple consequences for epithelial cells and may play a direct role in tumour cell invasion and cancer progression.

The role of MMPs in sE-cad production is further substantiated by Noe et al. [2000] who demonstrate increased release of sE-cad by MDCK kidney epithelial cells following treatment with recombinant SL-1, or matrilysin, another MMP. The presence of the MMP inhibitor TIMP-2 reduces levels of sE-cad in MCF-7 breast carcinoma cells. Purified and immobilized E-cadherin protein from both 


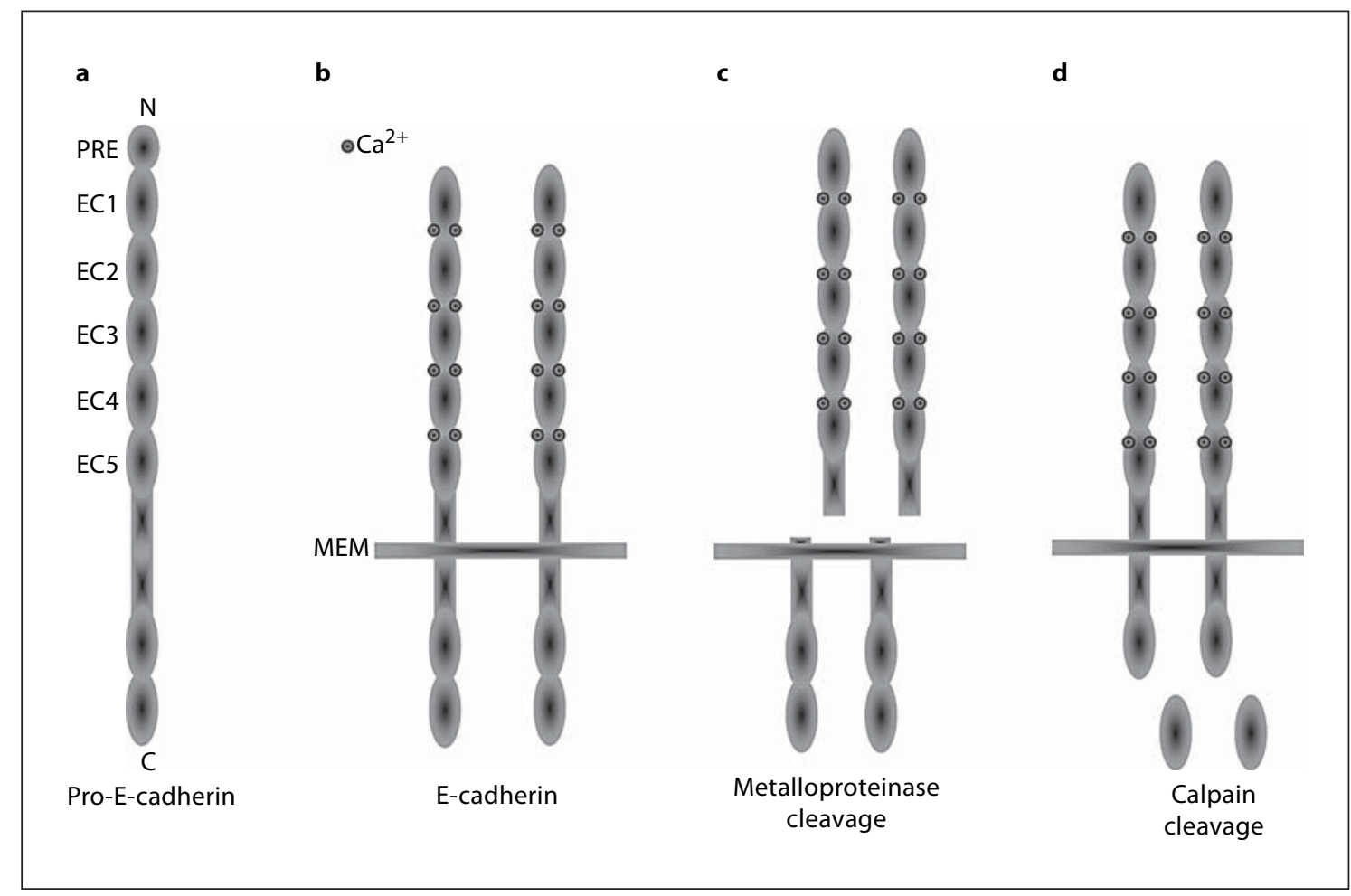

Fig. 1. Schematic diagram of E-cadherin and its posttranslational truncation products. a Pro-E-cadherin is cleaved near the $\mathrm{N}$-terminus by a subtilisin-like proprotein convertase removing a precursor $15-\mathrm{kDa}$ peptide (PRE). b Mature E-cadherin integrates into the cell membrane (MEM) and homodimers interact with dimers on adjacent epithelial cells. c Metalloproteinases cleave E-cadherin near the plasma membrane on the extracellular side. $\mathbf{d}$ Calpain cleaves E-cadherin upstream of the $\beta$ - and $\gamma$-catenin binding domains of the cytoplasmic tail.

MDCK and MCF-7 cells are directly cleaved by SL- 1 and matrilysin to yield 80 - and $40-\mathrm{kDa}$ fragments. Conditioned medium from SL-1- and matrilysin-treated MDCK cells inhibits cell aggregation and induces a more invasive phenotype. Thus the MMPs SL-1 and matrilysin can directly cleave E-cadherin to produce a soluble fragment that can increase invasion and inhibit cell aggregation in an autocrine manner.

Another protease implicated in the creation of truncated E-cadherin during tumour progression is calpain, a ubiquitously expressed protease involved in cell cycle regulation, signal transduction, cell migration and apoptosis. Calpain induces the accumulation of a $100-\mathrm{kDa}$ Ecadherin fragment $\left({\left.\mathrm{E}-\mathrm{cad}^{100}\right)}\right.$ in prostate LNCaP and breast MCF-7 epithelial cells (fig. 1d) [Rios-Doria et al., 2003]. Both $\mu$-and $\mathrm{m}$-calpain directly cleave E-cadherin to produce $\mathrm{E}$-cad ${ }^{100}$. The calpain cleavage site is located upstream of the $\beta$ - and $\gamma$-catenin binding domains of the cytoplasmic tail of E-cadherin, and amino acid residues
782-787 are essential for calpain-mediated cleavage. $\mathrm{m}$-Calpain expression is increased in localized and metastatic prostate cancer compared to benign prostate tumours and correlates with the appearance of E-cad ${ }^{100}$ in the tumour aspect of prostate tissue when compared to normal tissue. E-cad ${ }^{100}$ was also observed in all matched prostate metastases. Therefore, calpain expression is associated with prostate tumourigenesis, and calpain-mediated cleavage of E-cadherin is correlated with inactivation, specifically in prostate cancer and metastases [Rashid et al., 2001; Rios-Doria et al., 2003]. Calpain-mediated release of the catenin binding domain of E-cadherin may support the malignant transformation of these cells and contribute to destabilizing the adhesive function of E-cadherin.

Although proteolytic cleavage of E-cadherin has been associated with tumour progression, the mechanisms by which alternative forms of E-cadherin may facilitate carcinogenesis remain unclear. Hepatocyte growth factor/ 
scatter factor (HGF/SF) is a multifunctional cytokine that induces cell scattering in epithelial and cancer cells. HGF/SF treatment of LNCaPFGC prostate cancer cells increases $80-\mathrm{kDa}$ sE-cad secretion, reduces $\beta$-catenin binding capacity and increases cell scattering and invasive ability [Davies et al., 2001]. Inhibition of matrilysin prior to $\mathrm{HGF} / \mathrm{SF}$ treatment prevents $\mathrm{sE}$-cad production. This suggests that generation of an invasive phenotype in these cells in response to $\mathrm{HGF} / \mathrm{SF}$ treatment occurs via matrilysin activation.

A histidine-alanine-valine (HAV) amino acid sequence, crucial for homophilic cadherin interactions, is located in the extracellular domain of E-cadherin [Noe et al., 1999]. This sequence is highly conserved among cadherins and is therefore considered to have an important and specific function. Peptide constructs containing these HAV sequences induce epithelial cell invasion and reduce cell aggregation in epithelial cell lines in a cadherin-type and species-specific manner [Noe et al., 1999]. sE-cad may thus act in an autocrine manner to induce and support an invasive phenotype in cancer cells. HAV peptides increase expression of MMP-2, MMP-9 and MT1-MMP and enhance the invasive ability of 16HBE14obronchial epithelial cells [Nawrocki-Raby et al., 2003]. The increase in invasive potential is MMP dependent as indicated by its abrogation in the presence of MMP inhibitor. The HAV peptide which is conserved in all cad- herins may therefore play a role in MMP induction and subsequent cell invasion via the release of sE-cad from tumour cells.

These associations between E-cadherin truncations and cancer progression have stimulated exploration into the potential utility of truncated E-cadherin as a biomarker for cancer progression in the prostate [Kuefer et al., 2003]. Serum and urine levels of sE-cad, along with increased levels of MMPs, are potential indicators of metastatic progression in prostate cancer, and high levels of sE-cad may be an indicator of high recurrence levels of bladder cancer [Griffiths et al., 1996; Kuefer et al., 2003].

In summary, disruption of stable cell-cell adhesion is a fundamental process that facilitates proliferation, cell motility and tumour dissemination, and usually involves the loss of function of a number of proteins. We are currently investigating the role of E-cadherin inactivation via truncation during tumour progression. Signalling proteases such as stromelysin, matrilysin, calpain and $\mathrm{HGF} / \mathrm{SF}$ appear to be involved in this process, although precise mechanisms remain to be elucidated. The existence of truncated forms of E-cadherin and resulting effects on integrity of adherens junctions is an important consideration if E-cadherin is to be used as an indicator of tumour status.

\section{References}

Angst, B.D., C. Marcozzi, A.I. Magee (2000) The cadherin superfamily: diversity in form and function. J Cell Sci 114: 629-641.

Banks, R.E., W.H. Porter, P. Whelan, P.H. Smith, P.J. Selby (1995) Soluble forms of the adhesion molecule E-cadherin in urine. J Clin Pathol 48: 179-180.

Chaffer, C.L., E.W. Thompson, E.D. Williams (2007) Mesenchymal to epithelial transition in development and disease. Cells Tissues Organs 185: 7-19.

Damsky, C., J. Richa. D. Solter, K. Knudsen, C.A. Buck (1983) Identification and purification of a cell surface glycoprotein intracellular adhesion in embryonic and adult tissue. Cell 34: 455-466.

Davies, G., W.G. Jiang, M.D. Mason (2001) Matrilysin mediates extracellular cleavage of E-cadherin from prostate cancer cells: a key mechanism in hepatocyte growth factor/ scatter factor-induced cell-cell dissociation and in vitro invasion. Clin Cancer Res 7: 3289-3297.
Gofuku, J., H. Shiozaki, Y. Doki, M. Inoue, M. Hirao, N. Fukuchi, M. Monden (1998) Characterization of soluble E-cadherin as a disease marker in gastric cancer patients. Br J Cancer 78: 1095-1101.

Griffiths, T.R., I. Brotherick, R.I. Bishop, M.D. White, D.M. McKenna, C.H. Horne, B.K. Shenton, D.E. Neal, J.K. Mellon (1996) Cell adhesion molecules in bladder cancer: soluble serum E-cadherin correlates with predictors of recurrence. Br J Cancer 74: 579-584.

Hirano, S., A. Nose, K. Hatta, A. Kawakami, M. Takeichi (1987) Calcium-dependent cell-cell adhesion molecules (cadherins): subclass specificities and possible involvement of actin bundles. J Cell Biol 105: 2501-2510.

Ivanov, D.B., M.P. Philippova, V.A. Tkachuk (2001) Structure and functions of classical cadherins. Biochemistry 66: 1450-1464.

Katayama, M., S. Hirai, K. Kamihagi, K. Nakagawa, M. Yasumoto, I. Kato (1994) Soluble E-cadherin fragments increased in circulation of cancer patients. Br J Cancer 69: $580-$ 585.
Korinek, V., N. Barker, P.J. Morin, D. van Wichen, R. de Weger, K.W. Kinzler, B. Vogelstein, H. Clevers (1997) Constitutive transcriptional activation by a $\beta$-catenin-Tcf complex in $\mathrm{APC}^{-/-}$colon carcinoma. Science 275: 17841787.

Kuefer, R., M.D. Hofer, J.E. Gschwend, K.J. Pienta, M.G. Sanda, A.M. Chinnaiyan, M.A. Rubin, M.L. Day (2003) The role of an $80 \mathrm{kDa}$ fragment of E-cadherin in the metastatic progression of prostate cancer. Clin Cancer Res 9: 6447-6452.

Li, L., J. Backer, A.S.K. Wong, E.L. Schwanke, B.G. Stewart, M. Pasdar (2003) Bcl-2 expression decreases cadherin-mediated cell-cell adhesion. J Cell Sci 116: 3687-3700.

Lochter, A., S. Galosy, J. Muschler, N. Freedman, Z. Werb, M. Bissel (1997) Matrix metalloproteinase stromelysin-1 triggers a cascade of molecular alterations that leads to stable epithelial-to-mesenchymal conversion and a premalignant phenotype in mammary epithelial cells. J Cell Biol 139: 1861-1872. 
Nawrocki-Raby, B., C. Gilles, M. Polette, E. Bruyneel, J.Y. Laronze, N. Bonnet, J.M. Foidart, M. Mareel, P. Birembaut (2003) Upregulation of MMPs by soluble E-cadherin in human lung tumor cells. Int J Cancer 105: 790-795.

Noe, V., B. Fingleton, K. Jacobs, H.C. Crawford, S. Vermeulen, W. Steelant, E. Bruyneel, L.M. Matrisian, M. Mareel (2000) Release of an invasion promoter E-cadherin fragment by matrilysin and stromelysin-1. J Cell Sci 114: 111-118.

Noe, V., J. Willems, J. Vandekerckhove, F.V. Roy, E. Bruyneel, M. Mareel (1999) Inhibition of adhesion and induction of epithelial cell invasion by HAV-containing E-cadherin-specific peptides. J Cell Sci 112: 127-135.

Noritake, J., M. Fukata, K. Sato, M. Nakagawa, T. Watanabe, N. Izumi, S. Wang, Y. Fukata, K. Kaibuchi (2004) Positive role of IQGAP1, an effector of Racl, in actin-meshwork formation at sites of cell-cell contact. Mol Biol Cell 15: 1065-1076.

Ozawa, M., H. Baribault, R. Kemler (1989) The cytoplasmic domain of the cell adhesion molecule uvomorulin associates with three independent proteins structurally related in different species. EMBO J 8: 1711-1717.
Ozawa, M., R. Kemler (1990) Correct proteolytic cleavage is required for the cell adhesion function of uvomorulin. J Cell Biol 111: 1645-1650.

Peyrieras, N., F. Hyafil, D. Louvard, H.L. Ploegh, F. Jacob (1983) Uvomorulin: a nonintegral membrane protein of early mouse embryo. Proc Natl Acad Sci USA 80: 6274-6277.

Protheroe, A.S., R.E. Banks, M. Mzimba, W.H. Porter, J. Southgate, P.N. Singh, M. Bosomworth, P. Harnden, P. Smith, P. Whelan, P. Selby (1999) Urinary concentrations of the soluble adhesion molecule E-cadherin and total protein in patients with bladder cancer. Br J Cancer 80: 273-278.

Rashid, M.G., M.G. Sanda, C.J. Vallorosi, J. RiosDoria, M.A. Rubin, M.L. Day (2001) Posttranslational truncation and inactivation of human E-cadherin distinguishes prostate cancer from matched normal prostate. Cancer Res 61: 489-492.
Reinsch, S., E. Karsenti (1994) Orientation of spindle axis and distribution of plasma membrane proteins during cell division in polarized MDCKII cells. J Cell Biol 126 1509-1526.

Rios-Doria, J., K.C. Day, R. Kuefer, M.G. Rashid, A.M. Chinnaiyan, M.A. Rubin, M.L. Day (2003) The role of calpain in the proteolytic cleavage of E-cadherin in prostate and mammary epithelial cells. J Biol Chem 278: 13721379.

Shen, X., R.H. Kramer (2004) Adhesion-mediated squamous cell carcinoma survival through ligand-independent activation of epidermal growth factor receptor. Am J Pathol 165: 1315-1329.

Steinhusen, U., J. Weiske, V. Badock, R. Tauber, K. Bommert, O. Huber (2001) Cleavage and shedding of E-cadherin after induction of apoptosis. J Biol Chem 276: 4972-4980.

Wheelock, M.J., C.A. Buck, K.B. Bechtol, C.H Damsky (1987) Soluble 80-kd fragment of cell-CAM 120/80 disrupts cell-cell adhesion. J Cell Biochem 34: 187-202.

Zhu, W., B. Leber, D.A. Andrews (2001) Cytoplasmic O-glycosylation prevents cell surface transport of E-cadherin during apoptosis. EMBO J 20: 5999-6007. 
Copyright: S. Karger AG, Basel 2007. Reproduced with the permission of S. Karger AG, Basel. Further reproduction or distribution (electronic or otherwise) is prohibited without permission from the copyright holder. 